\title{
Masses and Magnetic Fields of White Dwarfs in Cataclysmic Variables
}

\author{
J.P. LASOTA ${ }^{1}$, J.M. HAMEURY ${ }^{2}, A \cdot R \cdot \mathrm{KING}^{1,2,3}$ \\ ${ }^{1}$ DARC, Observatoire de Paris, Section de Meudon, F-92195 Meudon Principal cédex, France \\ ${ }^{2}$ DAEC, Observatoire de Paris, Section de Meudon, F-92195 Meudon Principal cédex, and Uni- \\ versité Paris 7, France \\ ${ }^{3}$ Astronomy Department, University of Leicester, Leicester LE17RH, UK
}

\section{Summary}

We show that the existence of the AM Her period spike implies (i) a unique white dwarf mass $\simeq 0.6-0.7 M_{\odot}$ for most magnetic CV's (ii) nova explosions remove exactly the accreted mass from magnetic white dwarfs, and (iii) the maximum magnetic field for most CV's is $\leq 4 \times 10^{7} \mathrm{G}$. The existence of the spike is very strong support for the idea that the period gap results from a drastic reduction of angular momentum losses when the secondary star becomes fully convective.

\section{Introduction}

The period distribution of AM Herculis magnetic cataclysmic binaries is well known to be highly non-uniform. In particular, there is a large, highly significant ${ }^{1}$ accumulation (6 out of 15) of systems in the interval 113.5 - 114.8 minutes. Recently we (Hameury et al. ${ }^{2}$, hereafter HKLR) proposed an explanation for this in terms of the resumption of mass transfer after these systems have crossed the period gap. When accretion resumes at a period of about $2 \mathrm{hr}$, the fully convective secondary star undergoes a short (Kelvin-Helmholtz time) episode of adiabatic expansion, until it is sufficiently far out of thermal equilibrium that it can contract under the influence of mass loss. Therefore, the orbital period first increases by 2 to $3 \mathrm{~min}$, reaches a maximum, and decreases. This produces two effects which combine to enhance the discovery probability at this point: (i) the mass transfer rate $-\dot{M}_{2}$ is about twice the value for a star close to the main sequence contracting in response to mass loss, and (ii) the expansion and subsequent contraction of the secondary lengthens the time spent near the initial period. To give the observed period "spike" requires most systems to have similar parameters when mass transfer resumes at the lower edge of the period gap: otherwise the initial value of $P_{0}$ would not be the same and the spike would be smeared out. The secondary stars are naturally rather similar if, as suggested by Rappaport et al. $^{3}$ and Spruit \& Ritter ${ }^{4}$, the gap is caused by them becoming fully convective. Thus at the spike, $M_{2} \simeq 0.2 \mathrm{M}_{\odot}$. Further, the period spike implies constraints on the primary masses which have been examined by Hameury et $a .^{5}$ (hereafter HKL). Higher masses cause a narrower period gap and hence a higher value $P_{0}$ of the initial period below the gap. The discovery of EXO 0333192554.2 at a period of 127 min implies a significantly larger white dwarf mass (unless it is one 
of the rare systems which form in the period gap). This prediction was subsequently supported by radial-velocity measurements ${ }^{6}$. Thus, contrary to the suggestion of Berriman \& $\mathrm{Smith}^{7}$, the existence of an AM Her system at 127 min does not necessitate a modification of our model, but actually provides strong confirmation of its validity. In two recent papers ${ }^{8,9}$ we have examined the implications of the values of $M_{1}$ predicted for the spike and EXO 033319-2554.2 for the underlying mass distribution, nova efficiency and magnetic fields.

\section{Mass loss, secular evolution and nova efficiency.}

HKL calculate the secular evolution of CV's in which a fraction $\beta$ of the transferred mass is ultimately retained by the white dwarf, i.e. the primary and secondary masses vary with time according to:

$\frac{d M_{1}}{d t}=-\beta \frac{d M_{2}}{d t}$

Thus $\beta<0$ implies that nova explosions gradually reduce the mass of the white dwarf despite the accretion. The evolution is assumed to be driven by angular momentum losses via gravitational radiation and magnetic braking. The secondary is modelled as two polytropes (convective envelope and radiative core) after the prescription of Rappaport et $\mathrm{al}^{3}$. (see HKLR for full details). HKL consider the cases $\beta=0, \pm 0.5$ and $\beta=2 e^{-M_{1}^{4}}-1$, the latter describing situations in which mass ejection is much stronger in high mass systems. In each case it was required that the period $P_{0}$ at which the systems resume mass transfer after crossing the gap be $115 \mathrm{~min}$. The resulting values of $M_{1}$ for systems in the spike and EXO 033319-2554.2 are shown in table 1:

\begin{tabular}{|c|cccc|}
\hline$\beta$ & $M_{1}$ (spike) $/ \mathrm{M}_{\odot}$ & $M_{1}(\mathrm{EXO}) / \mathrm{M}_{\odot}$ & $P_{\max }(\mathrm{hr})$ & $P_{i n, \min }(\mathrm{hr})$ \\
\hline 0 & $0.70 \pm 0.10$ & $1.27 \pm 0.12$ & 7.2 & 4.0 \\
-0.5 & $0.48 \pm 0.13$ & $1.0 \pm 0.2$ & 10 & - \\
0.5 & $0.80 \pm 0.01$ & $1.43 \pm 0.01$ & 5 & - \\
$2 e^{-M_{1}^{4}-1}$ & $0.80 \pm 0.01$ & $1.09 \pm 0.01$ & 4.5 & - \\
\hline
\end{tabular}

Table 1: required masses of the primary and secondary for different values of $\beta$. It has been assumed (i) that the initial secondary mass is $0.6 \mathrm{M}_{\odot}$ and (ii) that the spike in the period distribution is at $115 \mathrm{~min}$ exactly; the range in $M_{1}$ does not take into account the width of the spike. This adds $\sim 0.05 \mathrm{M}_{\odot}$ to the uncertainties in $M_{1} . P_{\max }$ is the maximum period for stable mass transfer of a system which would resume mass transfer at a period of $114 \mathrm{~min}$, assuming that the secondary is on the initial main sequence when it first enters into contact (in this case, the secondary mass is not fixed at $0.6 \mathrm{M}_{\odot}$ ). $P_{i n, m i n}$ is the shortest period at which a system can form if the existence of the spike is not to require a relation between $M_{1}$ and $M_{2}$.

Systems with initial primary masses $M_{1, \text { in }}$ can in principle form at any period $P_{\text {in }}$ less than $P_{m a x}$, defined as the maximum period for which mass transfer is stable, assuming that the secondary is on the main sequence. At periods $\geq 4 \mathrm{hr}$, the systems would be intermediate polars ${ }^{10,11} . P_{\text {in }}$ and the initial secondary mass are related by the usual Roche lobe condition: ${ }^{12}$ 
$P_{\text {in }} \simeq 9 M_{2, \text { in }} / \mathrm{M}_{\odot} \mathrm{hr}$

In all cases except $\beta=0$ however, $M_{1, \text { in }}$ must be tightly specified as a function of $M_{2, \text { in }}$ if the subsequent evolution is to pass through the spike (i.e. resume mass transfer) at the correct period $P_{0} \simeq 114 \mathrm{~min}$. This is a direct consequence of the fact that if $\beta \neq 0$, the value of $M_{1}$ at a given period depends not only on $M_{1, \text { in }}$, but also on how much mass the white dwarf has lost or gained during the subsequent evolution, and hence for how long mass transfer has been running. The actual value of $P_{0}$ is very sensitive to the value of the mass ratio $M_{2} / M_{1}$ at the upper edge of the gap. This can be understood by realizing that the gap occurs because during the mass transer phase the secondary is out of equilibrium and therefore oversized with respect to its main sequence radius. When the braking mechanism is suddenly interrupted (or severely reduced) the secondary star contracts towards its main sequence configuration. The length of the gap will depend on how far its radius was from the main sequence value. For a given braking mechanism, this in turn depends on the mass ratio $M_{1} / M_{2}$. Unless $\beta \neq 0$ there must therefore be an unexplained relation between the initial $M_{1, i n}$ and $M_{2, \text { in }}$. We deduce that $\beta$ has to be very close to zero. Even for $\beta=0$ a system may miss the spike if it was born too close to the upper edge of the gap, and consequently the secondary did not have enough time to build up the required deviation from thermal equilibrium. One has then a minimum initial period $P_{i n, \min }=4 \mathrm{hr}$ and a minimum initial secondary mass $M_{2, \text { in }}=0.45 \mathrm{M}_{\odot}$.

The conclusion that $\beta \simeq 0$ is supported by nova models ${ }^{9}$ : in magnetic systems the field prevents mixing and therefore does not allow for the ejection of more mass than the amount accreted, but the white dwarf loses almost all the accreted material during the optically thick wind phase of the outburst.

The analysis of initial mass distribution and selection effects ${ }^{8}$ shows that strongly magnetic CV's can be divided into two groups:

1. the low $\left(\simeq 0.7 \mathrm{M}_{\odot}\right)$ mass group which accounts for $\sim 90 \%$ of observed magnetic CV's and 90-99\% of all magnetic CV's, and 2. high mass, with white dwarf masses in the range 1-1.4 $\mathrm{M}_{\odot}$;EXO 033319-2554.2 and $\mathrm{V} 1500$ Cyg are the two known members of this class.

\section{Magnetic field strengths}

Most AM Her binaries have white dwarfs magnetic fields ${ }^{13} B_{1} \leq 3 \times 10^{7} \mathrm{G}^{13}$, whereas isolated white dwarfs can have fields up to at least ${ }^{14} 5 \times 10^{8} \mathrm{G}$. Since most properties of magnetic binaries depend on the magnetic moment, the upper limit on magnetic field was difficult to understand. However, if one neglects the high mass group we may regard AM Her systems as having effectively a unique white dwarf mass. With $R_{1}$ essentially fixed at $\simeq 8 \times 10^{8} \mathrm{~cm}$, the limit on $\mathrm{B}$ becomes a limit on $\mu_{1}$ through $\mu_{1}=5 \times 10^{33} B_{7} \mathrm{G} \mathrm{cm}^{3}$ where $B_{7}$ is $B_{1}$ in units of $10^{7} \mathrm{G}$. We have shown ${ }^{9}$ that, $\mu_{1} \geq 2 \times 10^{34} \mathrm{G} \mathrm{cm}^{3}$, (thus, fields stronger than $B_{7} \simeq 4$ ) implies catastrophic angular momentum losses $\dot{J}$ as a result of coupling of the secondary stellar wind with the white dwarf magnetic field ${ }^{13}$. These drive the secular evolution so rapidly that the systems enter a prolonged period gap at $P \sim 5 \mathrm{hr}$, remaining as detached systems for a time of order the Hubble time. Some of these systems may in fact be observed as apparently isolated, rapidly rotating and highly magnetic white dwarfs ${ }^{14}$, or as non-magnetic white dwarfs with brown dwarf companions. 


\section{Conclusions}

The AM Her period spike implies that:

(i) the period of $114 \mathrm{~min}$ is special: all secondaries are identical at the lower edge of the gap, (ii) $M_{1} \simeq 0.6-0.7 M_{\odot}$ for most systems, (iii) $M_{1}$ stays constant during the evolution; nova outbursts on magnetic CV's eject just the accreted matter, (iv) the mass of EXO 033319-2554.2, with a period of $127 \mathrm{~min}$ is $\simeq 1.3 M_{\odot}$, (v) for most systems $B_{\max } \leq 4 \times 10^{7} \mathrm{G}$.

\section{References}

1. Morris, S.L., Schmidt, G.D., Liebert, J., Stocke, J., Gioia, I. \& Maccacaro, T., 1987. Astrophys. $J ., 314,641$.

2. Hameury, J.M., King, A.R., Lasota, J.P. \& Ritter, H., 1988a. Mon. Not. R. astr. Soc., 231, 535.

3. Rappaport, S., Verbunt, F. \& Joss, P.C., 1983. Astrophys. J., 275, 713.

4. Spruit, H.C. \& Ritter, H., 1983. Astr. Astrophys., 124, 267.

5. Hameury, J.M., King, A.R. \& Lasota, J.P., 1988b. Astr. Astrophys., 195, L12.

6. Beuermann, K., Thomas, H.C. \& Schwope, A., 1988. Astr. Astrophys., 195, L15.

7. Berriman, G. \& Smith, P.S., 1988. Astrophys. J., 329, L97.

8. Hameury, J .M., King, A.R., Lasota, J.P. \& Livio, M., 1988c. Mon. Not. R. astr. Soc., , .submitted

9. Hameury, J .M., King, A.R. \& Lasota, J.P., 1988d. Mon. Not. R. astr. Soc., , submitted

10. King, A.R., Frank, J. \& Ritter, H., 1985. Mon. Not. R. astr. Soc., 213, 185.

11. Hameury, J .M., King, A.R. \& Lasota, J.P., 1986. Mon. Not. R. astr. Soc., 218, 695.

12. Warner, B., 1976. in Structure and evolution of close binary systems, IAU symposium No 73, eds. P. Eggleton, S. Mitton and J. Whelan, Reidel, Dordrecht, Holland, p. 85.

13. Schmidt, G., Stockman, H.S. \& Grandi, S.A., 1986. Astrophys. J., 300, 804.

14. Schmidt, G., 1987. Mem. Soc. Astron. It. 209, 227. 